On achievable rate regions of the asymmetric AWGN two-way relay channel

by Lawrence Ong, Christopher M. Kellett, \& Sarah J. Johnson

Copyright (C) 2011 IEEE.

This is an author-prepared version of the article, reprinted from IEEE International Symposium on Information Theory Proceedings, ISIT 2011, p. 84-88.

http://dx.doi.org/10.1109/ISIT.2011.6034256

This material is posted here with permission of the IEEE. Such permission of the IEEE does not in any way imply IEEE endorsement of any of University of Newcastle's products or services. Internal or personal use of this material is permitted. However, permission to reprint/republish this material for advertising or promotional purposes or for creating new collective works for resale or redistribution must be obtained from the IEEE by writing to pubs-permissions@ieee.org. By choosing to view this document, you agree to all provisions of the copyright laws protecting it. 


\title{
On Achievable Rate Regions of the Asymmetric AWGN Two-Way Relay Channel
}

\author{
Lawrence Ong, Christopher M. Kellett, and Sarah J. Johnson \\ School of Electrical Engineering and Computer Science, The University of Newcastle, Australia \\ Email: lawrence.ong@cantab.net, \{chris.kellett, sarah.johnson\}@ newcastle.edu.au)
}

\begin{abstract}
This paper investigates the additive white Gaussian noise two-way relay channel, where two users exchange messages through a relay. Asymmetrical channels are considered where the users can transmit data at different rates and at different power levels. We modify and improve existing coding schemes to obtain three new achievable rate regions. Comparing four downlinkoptimal coding schemes, we show that the scheme that gives the best sum-rate performance is (i) complete-decode-forward, when both users transmit at low signal-to-noise ratio (SNR); (ii) functional-decode-forward with nested lattice codes, when both users transmit at high SNR; (iii) functional-decode-forward with rate splitting and time-division multiplexing, when one user transmits at low SNR and another user at medium-high SNR.
\end{abstract}

\section{INTRODUCTION}

We investigate the additive white Gaussian noise two-way relay channel (AWGN TWRC) depicted in Fig. 1. We modify existing coding schemes and obtain three new achievable rate regions. We compare these three modified coding schemes with an existing scheme, and show that different schemes give the best sum-rate performance for different SNR regions.

The AWGN TWRC we consider has no direct link between the users; data exchange between the users is done through a relay. The AWGN TWRC is defined by two AWGN channels: the uplink from the users to the relay, and the downlink from the relay to the users.

If we assume that a genie informs the relay of both users' messages, and only consider the downlink, i.e., how the relay sends these message, the downlink capacity region is known [1], [2]. However, the capacity region of the AWGN TWRC is unknown in general, and the main difficulty lies in determining the best way the relay should process its received signals on the uplink.

Knopp [3] proposed two coding schemes: (i) amplifyforward ${ }^{1}$ where the relay simply scales its received signals on the uplink and transmits them on the downlink, and (ii) complete-decode-forward ${ }^{2}$ (CDF) where the relay decodes both users' messages on the uplink, re-encodes and sends both messages on the downlink. Schnurr et al. [4] later proposed the compress-forward scheme where the relay quantizes its received signals on the uplink, re-encodes the quantized signals, and sends them on the downlink.

\footnotetext{
${ }^{1}$ This scheme was called analog relaying in [3], but is now commonly referred to as amplify-forward.

${ }^{2}$ This scheme was called digital relaying in [3], but is now commonly referred to as decode-forward. We term this scheme CDF to differentiate it from another scheme where the relay only decodes a function of the users' messages.
}

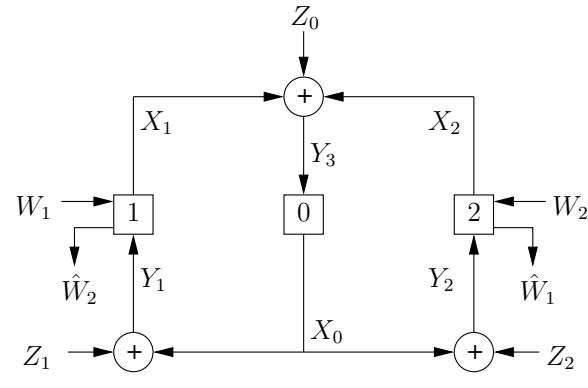

Fig. 1. The AWGN TWRC, where two users (nodes 1 and 2) exchange messages $\left(W_{1}\right.$ and $\left.W_{2}\right)$ through a relay (node 0 )

$\mathrm{CDF}$, where the relay removes the uplink noise, is downlink optimal in the sense that the downlink channel usage achieves the downlink capacity region ${ }^{3}$ [2]. On the other hand, in the amplify-forward and the compress-forward schemes, the uplink channel noise propagates to the downlink and hence they are not downlink optimal. In this paper, we will focus on coding schemes that are downlink optimal.

In CDF, after the relay decodes both users' messages, instead of sending both messages on the downlink, Kramer and Shamai [1] showed it is also downlink optimal for the relay to transmit only a function of the messages.

Instead of decoding the individual messages and transmitting only a function of the messages, the relay might directly decode this function on the uplink. We term this scheme functional-decode-forward (FDF). Obviously, the function must be defined such that on the downlink, each user can decode the message of the other user from the function and its own message. In addition, the channel code must match the uplink so that the relay can decode the codeword that carries the function of the users' messages without needing to decode the messages individually.

For the AWGN TWRC where both users transmit at the same power and at the same data rate, Narayanan et al. [5] proposed FDF using lattice $\operatorname{codes}^{4}$ (which are linear under the modulo-lattice operation) where the relay decodes a function, i.e., modulo-lattice summation, of the user's messages. This scheme approaches the capacity region of the AWGN TWRC asymptotically as the SNR grows. Using this scheme, both users transmit using the same lattice code and hence at the

\footnotetext{
${ }^{3}$ Decoding both users' messages is not always optimal for the uplink.

${ }^{4}$ Lattice codes have been shown to achieve the capacity of the point-to-point AWGN channel [6].
} 
same rate. For the asymmetrical case where the users transmit at different rates, Knopp [7] proposed a rate-splitting scheme as follows. The user with the lower rate transmits its message using a lattice code. The other user splits its message, and simultaneously transmits the sum (superposition) of (i) the first part of its message using the same lattice code, and (ii) the rest of the message using a random Gaussian code. This scheme introduces interference between the lattice codeword and the Gaussian codeword. To avoid this, Nam et al. [8], [9] used nested lattice codes, where one lattice code is a subset of the other lattice code, so the users transmit at different rates using lattice codes. However, this scheme suffers when there is a large difference between the users' transmit power levels.

The contributions of this paper are as follows:

(i) We improve the achievable rate region of FDF with nested lattice codes proposed by Nam et al. [9]. We note that for certain SNRs, if a user transmits at a lower (than the maximum allowable) power, the achievable rate of the other user can be increased, and the sum rate can also be increased.

(ii) We correct and improve the achievable rate region of FDF with rate splitting and simultaneous transmission proposed by Knopp [7]. When two users transmit using the same lattice code, and the relay decodes the modulolattice addition of the codewords, the achievable rate of $\frac{1}{2} \log (1+$ SNR $)$ used in [7] is incorrect. In addition, similar to FDF with nested lattice codes, we note that using less than the maximum allowable power, the achievable sum rate can be increased.

(iii) We propose a coding scheme using FDF with rate splitting and time-division multiplexing, and obtain a new achievable rate region. With rate splitting, one user transmits using a lattice code, while the other user uses the same lattice code and a Gaussian code. Instead of having the users transmit all codewords simultaneously, we split the transmission of the users into two phases: in the first phase, both users transmit the lattice codewords; in the second phase, one user transmits the Gaussian codeword.

(iv) As these schemes have the same downlink performanceall are downlink optimal-we compare their achievable sum rates on the uplink and obtain the following:

(a) In the low SNR region, CDF outperforms the other schemes.

(b) In the high SNR region, FDF with nested lattice codes outperforms the other schemes.

(c) When one user transmits at low SNR and the other user at medium-to-high SNR, FDF with rate splitting and time-division multiplexing outperforms the other schemes.

(d) For all SNRs, at least one of the three schemes(1) CDF, (2) FDF with nested lattice codes, or (3) FDF with rate splitting and time-division multiplexing-is able to outperform or match FDF with rate splitting and simultaneous transmission.

\section{Channel Model}

The AWGN TWRC depicted in Fig. 1 consists of three nodes: nodes 1 and 2 are the users, and node 0 the relay. We define by $X_{i}$ the transmitted signal of node $i$, and by $Y_{i}$ the received signal of node $i$. The AWGN TWRC is defined by the uplink channel $Y_{0}=X_{1}+X_{2}+Z_{0}$, and the downlink channel $Y_{i}=X_{0}+Z_{i}$, for $i \in\{1,2\}$. Each $X_{i}$ is subject to the power constraint $E\left[X_{i}^{2}\right] \leq P_{i}$, and each $Z_{i}$ is independent white Gaussian noise with power $E\left[Z_{i}^{2}\right] \leq N_{i}$, for $i \in\{0,1,2\}$. We say that users 1 and 2 transmit at SNRs equal to $P_{1} / N_{0}$ and $P_{2} / N_{0}$ respectively ${ }^{5}$. Consider $n$ simultaneous uplink and downlink channel uses, in which user 1 is to send an $n R_{1}$-bit message $W_{1}$ to user 2 , and user 2 is to send an $n R_{2}$-bit message $W_{2}$ to user 1 . In the $t$ th uplink channel use, each user $i$ transmits a function of its message and its previously received signals, i.e., $X_{i}[t]=$ $f_{i, t}\left(W_{i}, Y_{i}[1], Y_{i}[2], \ldots, Y_{i}[t-1]\right)$, for all $t \in\{1,2, \ldots, n\}$ and $i \in\{1,2\}$. In the $t$-th downlink channel use, the relay transmits a function of what it previously received, i.e., $X_{0}[t]=f_{0, t}\left(Y_{0}[1], Y_{0}[2], \ldots, Y_{0}[t-1]\right)$. After $n$ channel uses, user 1 produces an estimate of $W_{2}$ from its received messages and its own message, $\hat{W}_{2}=g_{1}\left(W_{1}, Y_{1}[1], Y_{1}[2], \ldots, Y_{1}[n]\right)$. User 2 does likewise to produce $\hat{W}_{1}$. The rate pair $\left(R_{1}, R_{2}\right)$ is said to be achievable if the probability of decoding error $\operatorname{Pr}\left\{\left(\hat{W}_{1}, \hat{W}_{2}\right) \neq\left(W_{1}, W_{2}\right)\right\}$ can be made as small as desired, with a sufficiently large $n$. The capacity region is the closure of all achievable rate pairs.

\section{EXISTING RESUlts}

\section{A. Capacity Outer Bound}

We define $C(x)=\frac{1}{2} \log (1+x)$. An outer bound to the capacity region of the AWGN TWRC is given as follows:

Theorem 1 ([7]): Consider an AWGN TWRC, a rate pair $\left(R_{1}, R_{2}\right)$ is achievable only if

$$
\begin{aligned}
& R_{1} \leq \min \left\{C\left(P_{1} / N_{0}\right), C\left(P_{0} / N_{2}\right)\right\} \\
& R_{2} \leq \min \left\{C\left(P_{2} / N_{0}\right), C\left(P_{0} / N_{1}\right)\right\} .
\end{aligned}
$$

The above outer bound can be obtained from the cut-set bound for the general multiterminal network $[10$, p. 589]. Together, the constraints $R_{1} \leq C\left(P_{0} / N_{2}\right)$ and $R_{2} \leq C\left(P_{0} / N_{1}\right)$ give the downlink capacity region, which only depends on the downlink channel parameters $P_{0}, N_{1}$, and $N_{2}$.

\section{B. Complete-Decode-Forward}

Using CDF, the following rate region is achievable:

Theorem 2: Consider an AWGN TWRC. CDF achieves the rate pair $\left(R_{1}, R_{2}\right)$ if

$$
\begin{aligned}
R_{1} & \leq C\left(P_{1} / N_{0}\right) \\
R_{2} & \leq C\left(P_{2} / N_{0}\right) \\
R_{1}+R_{2} & \leq C\left(\left[P_{1}+P_{2}\right] / N_{0}\right) \\
R_{1} & \leq C\left(P_{0} / N_{2}\right) \\
R_{2} & \leq C\left(P_{0} / N_{1}\right) .
\end{aligned}
$$

\footnotetext{
${ }^{5}$ Different SNRs here can be used to model different channel gains from the users to the relay. Varying $N_{1}$ and $N_{2}$ can be used to achieve the same effect on the channels from the relay to the users.
} 
The above region is obtained by porting the rate region of CDF for the half-duplex discrete memoryless TWRC [3] to the full-duplex AWGN TWRC. Using CDF, the encoding and decoding on the uplink are as follows:

\begin{tabular}{|l|l|}
\hline Codelength & $n$ \\
\hline User 1 & $\boldsymbol{U}_{1}\left(W_{1}\right)$ \\
\hline User 2 & $\boldsymbol{U}_{2}\left(W_{2}\right)$ \\
\hline Relay & decodes $W_{1}$ and $W_{2}$ \\
\hline
\end{tabular}

where $\boldsymbol{U}_{i} \in \mathcal{C}_{\text {Gaussian, } i}$ is the length-n Gaussian codeword transmitted by user $i, \mathcal{C}_{\text {Gaussian }, i}$ is the random Gaussian code for user $i$ with all codeletters independently generated according to the Gaussian distribution with $E\left[U_{i}^{2}\right]=P_{i}$. Here and in the rest of the paper, bold letters denote vectors.

The relay decodes both $W_{1}$ and $W_{2}$ on the uplink (i.e., a multiple-access channel), and it can reliably do so (i.e., with arbitrarily small decoding error) if (3)-(5) are satisfied [10, p. 526]. On the downlink, the relay sends $\left(W_{1}, W_{2}\right)$. Knowing its own message, each relay can reliably decode the message of the other user if (6) and (7) are satisfied [11].

\section{FUNCTIONAL-DECODE-FORWARD}

\section{A. FDF with Nested Lattice Codes}

We improve on the FDF with nested lattice codes scheme developed by Nam et al. [9] where both users transmit at their maximum allowable power. We modify the scheme such that user $i$ transmits at power $\delta_{i} P_{i}$ where $0 \leq \delta_{i} \leq 1$, for $i \in$ $\{1,2\}$, and obtain the following new achievable rate region.

Theorem 3: Consider the AWGN TWRC. FDF with nested lattice codes achieves the rate pair $\left(R_{1}, R_{2}\right)$ if

$$
\begin{aligned}
R_{1} & \leq\left[\frac{1}{2} \log \left(\frac{\delta_{1} P_{1}}{\delta_{1} P_{1}+\delta_{2} P_{2}}+\frac{\delta_{1} P_{1}}{N_{0}}\right)\right]^{+} \\
R_{2} & \leq\left[\frac{1}{2} \log \left(\frac{\delta_{2} P_{2}}{\delta_{1} P_{1}+\delta_{2} P_{2}}+\frac{\delta_{2} P_{2}}{N_{0}}\right)\right]^{+} \\
R_{1} & \leq C\left(P_{0} / N_{2}\right) \\
R_{2} & \leq C\left(P_{0} / N_{1}\right),
\end{aligned}
$$

for some $0 \leq \delta_{1}, \delta_{2} \leq 1$. Here $[x]^{+}=\max \{x, 0\}$.

Note that setting $\delta_{1}=\delta_{2}=1$ might not give the largest rate region as increasing $\delta_{1}$ decreases the RHS of (9), and increasing $\delta_{2}$ decreases the RHS of (8).

Without loss of generality, assume that $\delta_{1} P_{1} \geq \delta_{2} P_{2}$. Using FDF with nested lattice codes, denoted by $\mathcal{C}_{\text {lattice }, 1}$ and $\mathcal{C}_{\text {lattice }, 2}$, where $\mathcal{C}_{\text {lattice, } 2} \subseteq \mathcal{C}_{\text {lattice, } 1 \text {, the users transmit the following: }}$

\begin{tabular}{|l|l|}
\hline Codelength & $n$ \\
\hline User 1 & {$\left[\boldsymbol{V}_{1}\left(W_{1}\right)+\boldsymbol{D}_{1}\right] \bmod \Lambda_{1}$} \\
\hline User 2 & {$\left[\boldsymbol{V}_{2}\left(W_{2}\right)+\boldsymbol{D}_{2}\right] \bmod \Lambda_{2}$} \\
\hline Relay & decodes the function $\boldsymbol{F} \triangleq$ \\
& {$\left[\boldsymbol{V}_{1}\left(W_{1}\right)+\boldsymbol{V}_{2}\left(W_{2}\right)+\boldsymbol{K}\right] \bmod \Lambda_{1}$} \\
\hline
\end{tabular}

where $\boldsymbol{V}_{i}\left(W_{i}\right) \in \mathcal{C}_{\text {lattice, } i}$ is the length- $n$ lattice codeword for user $i$, the lattices $\Lambda_{1}$ and $\Lambda_{2}$ satisfy $\Lambda_{1} \subseteq \Lambda_{2}, D_{1}$ and $D_{2}$ are randomly generated length- $n$ dither vectors which are known to all nodes and are fixed for all transmissions, $\bmod \Lambda_{i}$ is the modulo-lattice operation [6], $\boldsymbol{K}$ is a deterministic function of
$\left(\boldsymbol{V}_{2}\left(W_{2}\right)+\boldsymbol{D}_{2}\right)$, and $\boldsymbol{K} \bmod \Lambda_{2}=0$. If (8)-(9) are satisfied, the relay can decode $\boldsymbol{F}$ reliably [9]. The relay then sends $\boldsymbol{F}$ on the downlink. If (10) and (11) are satisfied, both users can reliably decode $\boldsymbol{F}$. User 1 performs $\left(\boldsymbol{F}-\boldsymbol{V}_{1}\right) \bmod \Lambda_{2}$ to obtain $W_{2}$, and user 2 performs $\left(\boldsymbol{F}-\boldsymbol{V}_{2}-\boldsymbol{K}\right) \bmod \Lambda_{1}$ to obtain $W_{1}$.

\section{B. FDF with Rate Splitting and Simultaneous Transmission}

Next, we correct and modify the achievable rate region using FDF with rate splitting and simultaneous transmission proposed by Knopp [7] to obtain the following new rate region:

Theorem 4: Consider the AWGN TWRC where $P_{1} \geq P_{2}$. FDF with rate splitting and simultaneous transmission achieves the rate pair $\left(R_{1}, R_{2}\right)$ if

$$
\begin{aligned}
R_{2} & \leq \frac{1}{2} \log \left(\frac{1}{2}+\frac{\eta_{2} P_{2}}{N_{0}+\eta_{1}\left(P_{1}-\eta_{2} P_{2}\right)}\right) \\
R_{1}-R_{2} & \leq C\left(\eta_{1}\left[P_{1}-\eta_{2} P_{2}\right] / N_{0}\right) \\
R_{1} & \leq C\left(P_{0} / N_{2}\right) \\
R_{2} & \leq C\left(P_{0} / N_{1}\right),
\end{aligned}
$$

for some $0 \leq \eta_{1}, \eta_{2} \leq 1$.

Without loss of generality, assume that $R_{1}>R_{2}$. Let $W_{1}=$ $\left[W_{1 a}, W_{1 b}\right]$ where $W_{1 a}$ contains $n R_{2}$ bits, and $W_{1 b}$ contains $n\left(R_{1}-R_{2}\right)$ bits. Two codes are generated: (i) a lattice code $\mathcal{C}_{\text {lattice }}$ and (ii) a random Gaussian code $\mathcal{C}_{\text {Gaussian. }}$. The uplink transmissions are as follows:

\begin{tabular}{|l|l|}
\hline Codelength & $n$ \\
\hline User 1 & {$\left[\boldsymbol{V}\left(W_{1 a}\right)+\boldsymbol{D}_{1}\right] \bmod \Lambda+\boldsymbol{U}\left(W_{1 b}\right)$} \\
\hline User 2 & {$\left[\boldsymbol{V}\left(W_{2}\right)+\boldsymbol{D}_{2}\right] \bmod \Lambda$} \\
\hline Relay & $\begin{array}{l}\text { decodes } \boldsymbol{G} \triangleq\left[\boldsymbol{V}\left(W_{1 a}\right)+\boldsymbol{V}\left(W_{2}\right)\right] \bmod \Lambda \\
\text { and then decodes } \boldsymbol{U}\left(W_{1 b}\right)\end{array}$ \\
\hline
\end{tabular}

where $\boldsymbol{V}\left(W_{1 a}\right), \boldsymbol{V}\left(W_{2}\right) \in \mathcal{C}_{\text {lattice }}$ are length- $n$ lattice codewords from the same lattice code, $\boldsymbol{U}\left(W_{1 b}\right) \in \mathcal{C}_{\text {Gaussian }}$ is the length- $n$ Gaussian codeword, and $\boldsymbol{D}_{1}$ and $\boldsymbol{D}_{2}$ are randomly generated length- $n$ dither vectors which are known to all nodes and are fixed for all transmissions.

The relay first decodes $\boldsymbol{G}$ by treating $\boldsymbol{U}$ as noise, subtracts $\boldsymbol{G}$ off its received signals, and then decodes $\boldsymbol{U}$. The relay then sends $(\boldsymbol{G}, \boldsymbol{U})$ on the downlink. The above scheme was proposed in [7]. We make the following modifications:

- We note that the users might not transmit at their full available power, as the power used by user 1 to transmit $\boldsymbol{U}$ acts as an interference when the relay decodes $\boldsymbol{G}$. We propose that both users use $\eta_{2} P_{2}$ to transmit $\boldsymbol{V}$. User 1 then uses a fraction $\eta_{1}$ of its remaining power of $\left(P_{1}-\eta_{2} P_{2}\right)$ to transmit $\boldsymbol{U}$.

- We correct a minor error in the rate region for $R_{2}$ reported in [7] (c.f. (12)). $R_{2}$ is the rate of the lattice code used by both users with the same power. The relay attempts to decode the modulo-lattice sum of the lattice codewords, i.e., $\boldsymbol{G}$, in the presence of channel noise of power $N_{0}$ and $\boldsymbol{U}$ of power $\eta_{1}\left(P_{1}-\eta_{2} P_{2}\right)$. It has been shown in [5], [12] that the relay can reliably decode the modulo sum of lattice codewords if $R_{2} \leq \frac{1}{2} \log \left(\frac{1}{2}+\mathrm{SNR}\right)$, where the SNR in our modified scheme is $\eta_{2} P_{2} /\left(N_{0}+\eta_{1}\left[P_{1}-\eta_{2} P_{2}\right]\right)$. Narayanan 
et al. [5] conjectured that the rate of $R_{2}=\frac{1}{2} \log (1+\mathrm{SNR})$ (reported in [7]) cannot in fact be achieved.

The relay can reliably decode $G$ if (12) is satisfied. The relay then removes $\boldsymbol{G}$ and can reliably decode $\boldsymbol{U}$ if (13) is satisfied. The relay then sends $(\boldsymbol{G}, \boldsymbol{U})$ on the downlink. Knowing $W_{1 b}$, user 1 removes $\boldsymbol{U}$ from its received signals, and it can decode $G$ if (15) is satisfied. It obtains $W_{2}$ from $G$ and $W_{1 a}$. If (14) is satisfied, user 2 can decode $(\boldsymbol{G}, \boldsymbol{U})$, from which it can obtain $W_{1}$.

Remark 1: It is also possible to decode the Gaussian codeword first by treating the lattice codewords as noise. However, deriving the rate expression for this scheme is difficult as the effective noise in this case is the sum of Gaussian noise and lattices. Similar difficulty is encountered when one attempts to derive the rate expression for simultaneous decoding at the relay, as lattice decoding (using ML decoding) is used to decode the modulo-lattice sum of the lattice codewords and typical set decoding is used to decode the Gaussian codeword.

Remark 2: This uplink scheme where a user simultaneously transmits lattice and Gaussian codes was also considered by Baik and Chung [13]. However, they employed a different coding scheme on the downlink, i.e., the relay's encoding.

\section{FDF with Rate Splitting and Time-Division Multiplexing}

Next, we propose another coding scheme by modifying the rate splitting scheme in Sec. IV-B, and obtain the following:

Theorem 5: Consider the AWGN TWRC where $P_{1} \geq$ $P_{2}$. FDF with rate splitting and time-division multiplexing achieves the rate pair $\left(R_{1}, R_{2}\right)$ if

$$
\begin{aligned}
R_{2} & \leq \frac{\alpha}{2} \log \left(\frac{1}{2}+\frac{P_{2}}{\alpha N_{0}}\right) \\
R_{1}-R_{2} & \leq(1-\alpha) C\left(\frac{P_{1}-P_{2}}{(1-\alpha) N_{0}}\right) \\
R_{1} & \leq C\left(P_{0} / N_{2}\right) \\
R_{2} & \leq C\left(P_{0} / N_{1}\right),
\end{aligned}
$$

for some $0 \leq \alpha \leq 1$.

Again, we assume that $R_{1}>R_{2}$, and we split the message $W_{1}=\left[W_{1 a}, W_{1 b}\right]$, where $W_{1 a}$ has $n R_{2}$ bits, and $W_{1 b}$ has $n\left(R_{1}-R_{2}\right)$ bits. We generate two codes: (i) a lattice code $\mathcal{C}_{\text {lattice }}$ with codewords of length $\alpha n$ each, and (ii) a random Gaussian code $\mathcal{C}_{\text {Gaussian }}$ with codewords of length $(1-\alpha) n$ each. In the first $\alpha n$ uplink channel uses, both users transmit using the same lattice code with power $P_{2} / \alpha$. In the next $(1-\alpha) n$ channel uses, user 1 transmits with the Gaussian code using its remaining power $\left(P_{1}-P_{2}\right) /(1-\alpha)$. The uplink encoding and decoding are as follows:

\begin{tabular}{|l|l|l|}
\hline Codelength & $\alpha n$ & $(1-\alpha) n$ \\
\hline User 1 & {$\left[\boldsymbol{V}\left(W_{1 a}\right)+\boldsymbol{D}_{1}\right] \bmod \Lambda$} & $\boldsymbol{U}\left(W_{1 b}\right)$ \\
\hline User 2 & {$\left[\boldsymbol{V}\left(W_{2}\right)+\boldsymbol{D}_{2}\right] \bmod \Lambda$} & - \\
\hline Relay & $\begin{array}{l}\text { decodes } \boldsymbol{G} \triangleq\left[\boldsymbol{V}\left(W_{1 a}\right)\right. \\
\left.+\boldsymbol{V}\left(W_{2}\right)\right] \bmod \Lambda\end{array}$ & $\operatorname{decodes} \boldsymbol{U}\left(W_{1 b}\right)$ \\
\hline
\end{tabular}

where $\boldsymbol{V}\left(W_{1 a}\right), \boldsymbol{V}\left(W_{2}\right) \in \mathcal{C}_{\text {lattice }}$ are length- $\alpha n$ lattice codewords from the same lattice code, $\boldsymbol{U}\left(W_{1 b}\right) \in \mathcal{C}_{\text {Gaussian }}$ is the length- $(1-\alpha) n$ Gaussian codeword, and $\boldsymbol{D}_{1}$ and $\boldsymbol{D}_{2}$ are randomly generated length- $\alpha n$ dither vectors which are known to all nodes and are fixed for all transmissions.

In the first $\alpha n$ channel uses, the relay decodes the summation of lattice codewords $G$. The relay can reliably decode $G$ if (16) is satisfied [5]. The next $(1-\alpha) n$ channel uses are AWGN point-to-point channel uses from user 1 to the relay without user 2's interference. So, the relay can reliably decode $W_{1 b}$ if (17) is satisfied. After the relay obtains $(\boldsymbol{G}, \boldsymbol{U})$, the downlink transmission is the same as that of FDF with rate splitting and simultaneous transmission. Hence, we get (18)-(19).

Remark 3: Our proposed scheme differs from FDF with rate splitting and simultaneous transmission in (at least) the following two ways:

(i) The lattice codes and the random Gaussian codes are of different lengths. The codelengths are proportional to the time fraction of the respective transmissions.

(ii) There is no interference between the lattice codewords and the Gaussian codeword.

Remark 4: The downlink constraints on the achievable rate regions of all four schemes discussed coincide with the downlink capacity region. So, these schemes are downlink optimal.

\section{Sum Rate COMPARISON}

In this section, we compare the sum rate $R_{\text {sum }} \triangleq R_{1}+$ $R_{2}$ of the four schemes described in the previous sections to the sum rate upper bound. Note that a larger sum rate does not necessarily mean the entire two-dimensional rate region is larger. We fix the uplink channel noise power $N_{0}=2$. Without loss of generality, we assume that $P_{1} \geq P_{2}>0$. For the case of $P_{2} \geq P_{1}$, we simply reverse the roles of the users.

As these four schemes are downlink optimal, we only compare their uplink performance. This can be done by setting the relay power to be sufficiently high such that the downlink constraints will always be satisfied.

We fix $P_{2}$ and plot the maximum $R_{\text {sum }}$ achievable by each scheme by varying $P_{1}$. In Fig. 2 , we constrain user 2 to transmit at low SNR. When the other user (user 1) also transmits at low SNR, CDF gives the best performance. Still keeping user 2's SNR low, when user 1 transmits at high SNR, FDF with rate splitting and time-division multiplexing outperforms the other schemes. From Fig. 3, when both users are transmitting at high SNR, FDF using nested lattice codes outperforms the other schemes.

Fig. 4 gives a summary of schemes that achieve the highest sum rate for different $P_{1}$ and $P_{2}$ normalized by $N_{0}$. We observe the following: (i) When both users transmit at high SNR, FDF with nested lattice codes outperforms other schemes. (ii) When both users transmit at low SNR, CDF is the preferred scheme. (iii) When one user transmits at a low SNR and the other user at medium-to-high SNR, FDF with rate splitting and time-division multiplexing achieves the highest sum rate. In the equal-SNR region where the three FDF schemes attain the highest sum rate (i.e., $P_{1} / N_{0}=P_{2} / N_{0} \geq 1.75$ ), the three schemes effectively reduce to the same scheme: both users transmit using only a lattice code (the same lattice code) at the 


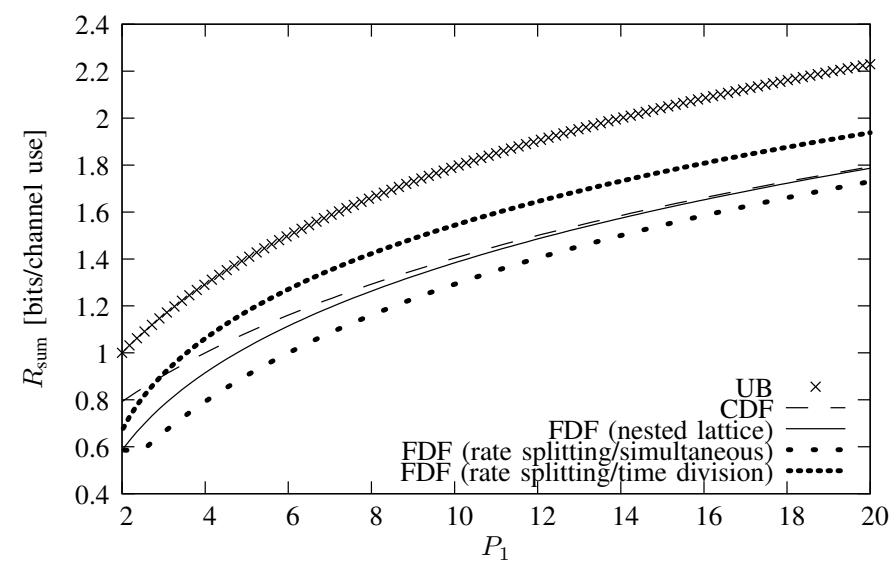

Fig. 2. Sum rate comparison, $N_{0}=2, P_{2}=2$

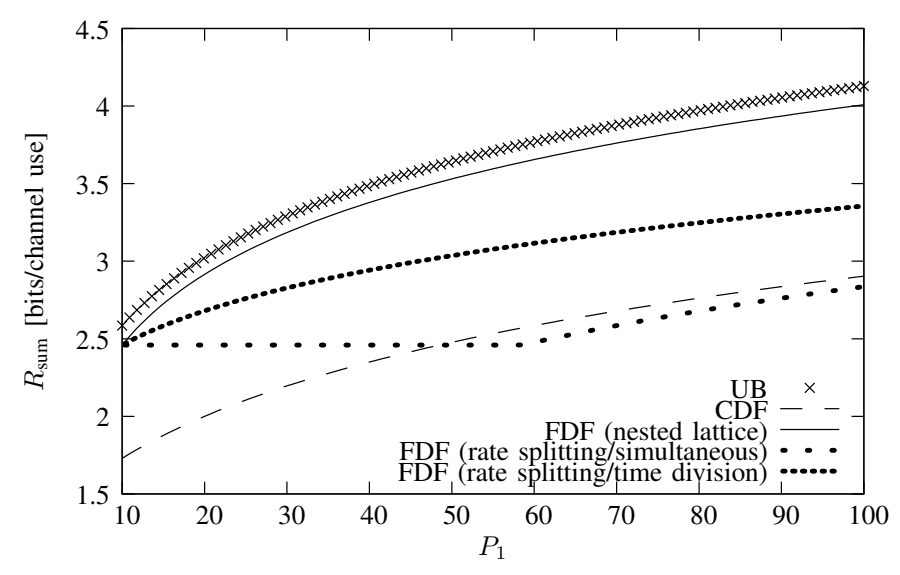

Fig. 3. Sum rate comparison, $N_{0}=2, P_{2}=10$

same power and no rate splitting is done. Also seen from the figure, FDF with rate splitting and simultaneous transmission does not give the (strictly) best sum rate at any SNR, i.e., one of the other schemes can always outperform or match it.

Using CDF, the relay needs to decode both the users' messages, c.f. (5). Because of the concavity of the $\log (\cdot)$ function, this constraint limits its performance at medium-to-high SNR. FDF, on the other hand, does not suffer from this problem. However, because of the modulo-lattice operation (see [14] for more discussion), FDF (which uses lattice codes) achieves rates up to $\frac{1}{2} \log (\gamma+\mathrm{SNR}$ ) where $\gamma<1$, while $\mathrm{CDF}$ (which uses Gaussian codes) achieves rate up to $\frac{1}{2} \log (1+\mathrm{SNR})$. So, FDF-based schemes do not perform well at low SNR. This explains why CDF performs better at low SNR, while FDFbased schemes perform better at medium-to-high SNR.

Using FDF with nested lattice codes, lattices of two different sizes (which depend on the transmit power) are used in the transmissions of the two users. As the relay decodes the modulo-sum of transmitted codewords with respect to the bigger lattice, the rate of the user that transmits using the smaller lattice (lower transmit power) is penalized. So, when one user transmits at high power and the other user at low power, the sum rates of FDF with nested lattice codes are affected; the sum rates of CDF are also affected by the reason

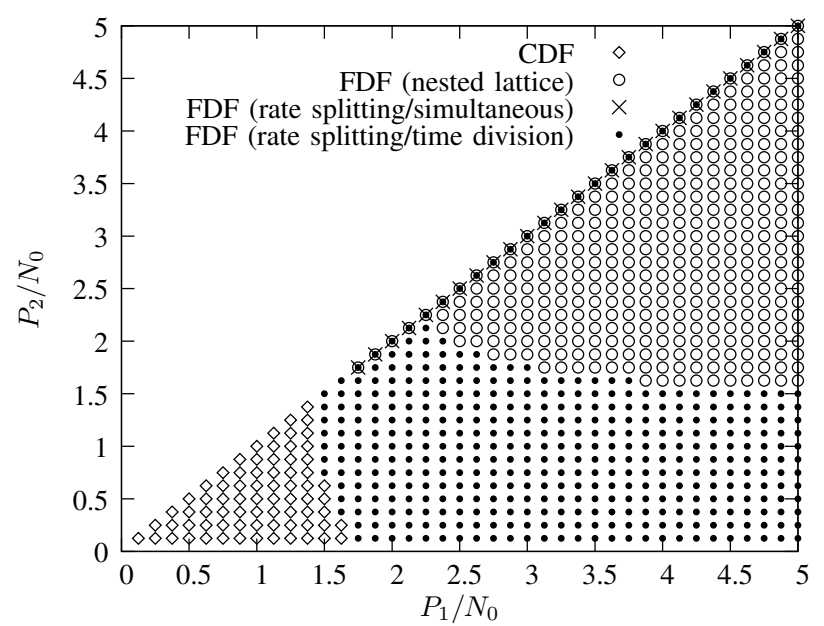

Fig. 4. Schemes that obtain the highest sum rate for varying $P_{1} / N_{0}$ and $P_{2} / N_{0}$

given in the previous paragraph. In this region, our proposed FDF with rate splitting and time-division multiplexing is able to give better sum-rates, as it does not suffer from the problems of the need to decode both users' messages and mismatched lattices.

\section{REFERENCES}

[1] G. Kramer and S. Shamai, "Capacity for classes of broadcast channels with receiver side information," in Proc. IEEE Inf. Theory Workshop (ITW), Lake Tahoe, USA, Sept. 2-6 2007, pp. 313-318.

[2] T. J. Oechtering, C. Schnurr, and H. Boche, "Broadcast capacity region of two-phase bidirectional relaying," IEEE Trans. Inf. Theory, vol. 54, no. 1, pp. 454-458, Jan. 2008.

[3] R. Knopp, "Two-way radio networks with a star topology," in Proc. Int. Zurich Semin. Commun. (IZS), Zurich, Switzerland, Feb. 22-24 2006, pp. 154-157.

[4] C. Schnurr, T. J. Oechtering, and S. Stanczak, "Achievable rates for the restricted half-duplex two-way relay channel," in Proc. 41st Asilomar Conf. Signal Syst. Comput., Pacific Grove, USA, Nov. 4-7 2007, pp. $1468-1472$.

[5] K. Narayanan, M. P. Wilson, and A. Sprintson, "Joint physical layer coding and network coding for bi-directional relaying," in Proc. 45th Allerton Conf. Commun. Control Comput. (Allerton Conf.), Monticello, USA, Sept. 26-28 2007, pp. 254-259.

[6] U. Erez and R. Zamir, "Achieving $\frac{1}{2} \log (1+\mathrm{SNR})$ on the AWGN channel with lattice encoding and decoding," IEEE Trans. Inf. Theory, vol. 50, no. 10, pp. 2293-2314, Oct. 2004.

[7] R. Knopp, "Two-way wireless communication via a relay station," in GDR-ISIS Meet., Paris, France, Mar. 292007.

[8] W. Nam, S. Chung, and Y. H. Lee, "Capacity bounds for two-way relay channels," in Proc. Int. Zurich Semin. Commun. (IZS), Zurich, Switzerland, Mar. 12-14 2008, pp. 144-147.

[9] - "Capacity of the Gaussian two-way relay channel to within $\frac{1}{2}$ bit," IEEE Trans. Inf. Theory, vol. 56, no. 11, pp. 5488-5494, Nov. 2010.

[10] T. M. Cover and J. A. Thomas, Elements of Information Theory, 2nd ed. Wiley-Interscience, 2006.

[11] E. Tuncel, "Slepian-Wolf coding over broadcast channels," IEEE Trans. Inf. Theory, vol. 52, no. 4, pp. 1469-1482, Apr. 2006.

[12] B. Nazer and M. Gastpar, "Lattice coding increases multicast rates for Gaussian multiple-access networks," in Proc. 45th Allerton Conf. Commun. Control Comput. (Allerton Conf.), Monticello, USA, Sept. 2628 2007, pp. 1089-1096.

[13] I. Baik and S. Chung, "Network coding for two-way relay channels using lattices," in Proc. IEEE Int. Conf. Commun. (ICC), Beijing, China, May 19-23 2008, pp. 3898-3902.

[14] U. Erez and R. Zamir, "A modulo-lattice transformation for multipleaccess channels," in Proc. 25th IEEE Conv. Electr. Electron. Eng. Israel, Tel Aviv, Israel, Dec. 3-5 2008, pp. 836-840. 